\section{Hospital-Acquired Infections Among Hemodialysis Patients}

The epidemiological characteristics of nosocomial infections among patients requiring chronic hemodialysis, a high-risk and rapidly growing population, have not been fully elucidated. D'Agata and coinvestigators conducted a 30-month cohort study and compared the rates of bloodstream infections, urinary tract infections (UTIs), pneumonia, and diarrhea caused by Clostridium difficile and the distribution of pathogens among hospitalized chronic hemodialysis patients to hospitalized patients not requiring chronic hemodialysis. To identify risk factors for developing a nosocomial infection among chronic hemodialysis patients, a matched case-control study was performed. A total of 1,557 nosocomial infections were detected during 1,317 ( $2 \%$ ) of 68,361 admissions. Of these, 47 nosocomial infections occurred in chronic hemodialysis patients during $31(5 \%)$ of 578 admissions.

Nosocomial infections were significantly more frequent among the chronic hemodialysis group $(9.1 / 1,000$ patient-days) compared with the non-chronic hemodialysis group (3.8/1,000 patient-days; relative risk [RR], 2.4; 95\% confidence interval $\left.\left[\mathrm{CI}_{95}\right], 1.8-3.2 ; P<.001\right)$. UTIs were the most common nosocomial infections among chronic hemodialysis patients, accounting for $47 \%$ of all infections in this population. UTIs were significantly more common among chronic hemodialysis patients $(4.2 / 1,000$ patientdays) compared with non-chronic hemodialysis patients (0.7/1,000 patient-days; RR, $\left.6.2 ; \mathrm{CI}_{95}, 3.8-9.5 ; P<.001\right)$. Among chronic hemodialysis patients, Candida species and enterococci were the most common pathogens in contrast to coagulase-negative staphylococci and Staphylococcus aureus among patients not requiring hemodialysis. Using conditional logistic regression, a greater index of comorbidity was significantly associated with nosocomial infections among the chronic hemodialysis population (odds ratio, 3.6; $\mathrm{CI}_{95}, 1.2-10.7 ; P=.02$ ).

Chronic hemodialysis patients were found to be at a substantially greater risk for developing a nosocomial infection compared with other hospitalized patients.

FROM: D'Agata EM, Mount DB, Thayer V, Schaffner W. Hospital-acquired infections among chronic hemodialysis patients. Am J Kidney Dis 2000;35:1083-1088.

\section{Outcomes of Elderly Patients With MRSA}

Morrison L. Stolarek, from the Department of Geriatric Medicine, Eastern General Hospital, Edinburgh, reported a retrospective study of admissions to the geriatric acute assessment and rehabilitation ward over a 1-year period to identify those with methicillin-resistant Staphylococcus aureus (MRSA) and determine whether this affected outcomes. Of 204 patients, 238 admissions were analyzed, and $9.8 \%$ of patients were MRSA positive. Demographics did not differ between MRSA-positive and -negative patients. Respiratory co-morbidity was more com- mon in MRSA-positive patients. Rates of functional decline did not differ between the two groups. Those colonized or infected by MRSA had a significantly longer stay (51.4 vs 32.2 days, $P=.03$ ), perhaps due to isolation and limited rehabilitation. The virulence of MRSA may be less in these patients, therefore isolation may be inappropriate and counterproductive

FROM: Stolarek ML. Does MRSA affect patient outcomes in the elderly? A retrospective pilot study. $J$ Hosp Infect 2000;45:169-171.

\section{Microbial Contamination and Connector Type}

Needleless connectors (NCs), which allow direct access to intravascular catheters, are widely used in clinical practice. The benefits of these devices to healthcare workers are well documented; however, the potential risk of microbial contamination and associated infection is unclear. Seymou and coinvestigators, from Birmingham, United Kingdom, conducted a study to evaluate microbial contamination rates for an $\mathrm{NC}$ as compared to a conventional three-way tap, which was connected to the hubs of central venous catheters (CVCs) immediately following insertion. Patients in the study group had NCs attached to the three-way taps, whereas the control group had standard entry port caps. On removal (up to 72 hours), the connectors were studied for microbial contamination.

There was no significant difference between the number of three-way taps contaminated on the internal surface with microorganisms in the control group with entry port caps $(19 / 132,14 \%)$ compared to the group with NCs $(18 / 105,17 \%)$. Sixteen percent $(27 / 173)$ of the NCs were contaminated with microorganisms on the internal surfaces. The external surface of $33 \%(27 / 82)$ of the NC silicone seals was contaminated after clinical use. Microorganisms were also isolated from 9\% (8/91) of the silicone seals after disinfection. The use of this needleless connector compared to standard caps therefore does not appear to increase the risk of infection via the internal lumen of three-way taps.

FROM: Seymou VM, Dhallu TS, Mos HA, Tebb SE, Elliot TS. A prospective clinical study to investigate the microbial contamination of a needleless connector. $J$ Hosp Infect 2000;45:165-168.

\section{Hydrogel- and Silver Salt-Coated Urinary Catheters and Incidence of UTI}

Catheters coated with hydrogel and silver salts have been proposed to prevent hospital-acquired urinary tract infections (UTIs). Thibon and colleagues, from Service d' Hygiene Hospitaliere, CHU de Caen, France, carried out a randomized, prospective, double-blinded, multicenter trial to compare those catheters with classical urinary tract catheters. They included in the study 199 patients requiring urethral catheterization for more than 3 days: 109 in 